УДК 621.396

\title{
ОЦЕНКА АМПЛИТУДЫ ПРЯМОУГОЛЬНОГО УЗКОПОЛОСНОГО РАДИОИМПУЛЬСА С НЕИЗВЕСТНЫМИ ДЛИТЕЛЬНОСТЬЮ И НАЧАЛЬНОЙ ФАЗОЙ“
}

\author{
А. П. ТРИФОНОВ, Ю. Э. КОРЧАГИН, М. Б. БЕСПАЛОВА, М. В. ТРИФОНОВ \\ Воронежский государственный университет, \\ Россия, Воронеж, 394006, Университетская пл., д. 1
}

\begin{abstract}
Аннотация. В работе исследованы алгоритмы оценки амплитуды радиосигнала с огибающей прямоугольной формы и с неизвестными длительностью и начальной фазой. Выполнены синтез и анализ квазиправдоподобного квазикогерентного алгоритма оценки, согласно которому вместо неизвестных длительности и начальной фазы используются некоторые ожидаемые их значения. Исследован проигрыш в точности оценки амплитуды вследствие априорного незнания длительности и начальной фазы. Синтезирован квазиправдоподобный некогерентный алгоритм оценки амплитуды с адаптацией начальной фазы и найдены его статистические характеристики: смешение и рассеяние оценки. Получены зависимости проигрыша в точности оценки вследствие априорного незнания длительности сигнала. Выполнены синтез и анализ максимально правдоподобного алгоритма оценки амплитуды, согласно которому вместо неизвестных длительности и начальной фазы используются их оценки максимального правдоподобия. Исследован выигрыш в точности максимально правдоподобной оценки по сравнению с квазиправдоподобными. Показано, что априорное незнание длительности сигнала асимптотически при больших отношениях сигнал/шум не влияет на точность максимально правдоподобной оценки амплитуды.
\end{abstract}

Ключевые слова: оценка амплитуды; неизвестные длительность и фаза; характеристики оценок амплитуды радиосигнала

Задача оценки амплитуды сигнала, наблюдаемого на фоне шума, представляет интерес для многих практических приложений радиоэлектроники и неоднократно рассматривалась в литературе [1-9]. В [1] рассмотрена оценка амплитуды детерминированного сигнала при условии, что все остальные параметры априори известны, найдены характеристики оценки амплитуды. В [2] исследована оценка максимального правдоподобия (МП) амплитуды сигнала, содержащего неизвестные неэнергетические параметры, а также совместные оцен- ки амплитуды и длительности прямоугольного импульса.

Однако широкое применение в радиоэлектронике находят также сигналы с высокочастотным заполнением (радиосигналы). Поэтому оказывается актуальной задача оценки амплитуды радиосигнала с неизвестными длительностью и начальной фазой высокочастотного заполнения. Ниже рассмотрены алгоритмы оценки амплитуды узкополосного радиоимпульса с прямоугольной формой огибаю-

* Исследование выполнено за счёт гранта Российского научного фонда (проект 15-11-10022).

DOI: $10.20535 / \mathrm{S} 0021347017120019$

๑) А. П. Трифонов, Ю. Э. Корчагин, М. Б. Беспалова, М. В. Трифонов, 2017 


\section{БИБЛИОГРАФИЧЕСКИЙ СПИСОК}

1. Тихонов, В.И. Оптимальный прием сигналов. М.: Радио и связь, 1983. 320 с.

2. Трифонов, А.П.; Шинаков, Ю.С. Совместное различение сигналов и оченка их параметров на фоне помех. М.: Радио и связь, 1986. 268 с.

3. Трифонов, А.П.; Корчагин, Ю.Э.; Кондратович, П. А.; Трифонов, М.В. Оценка амплитуды сигнала 
с неизвестной длительностью. Известия вузов. Радиоэлектроника, Т. 55, № 9, C. 3-10, 2012. URI: http://radio.kpi.ua/article/view/S0021347012090014.

4. Зандер, Ф. В.; Чмых, М.К.Предельные погрешности оптимальных измерителей амплитуды и постоянной составляющей сигналов с малым временем измерения. Измерительная техника, № 1, С. 33-34, 1988.

5. Wang, Chunyang; Liu, Xuelian; Fan, Bin. Estimation method for weak sinusoidal amplitude in alpha noise. Proc. of 12th Int. Conf. on Signal Processing, ICSP, 19-23 Oct. 2014, Hangzhou, China. IEEE, 2014, pp. 46-51. DOI: 10.1109/ICOSP.2014.7014967.

6. Czuszynski, Krzysztof; Ruminski, Jacek; Polinski, Artur; Bujnowski, Adam. Estimation of the amplitude of the signal for the active optical gesture sensor with sparse detectors. Proc. of 9th Int. Conf. on Human System Interactions, HSI, 6-8 Jul. 2016, Portsmouth, UK. IEEE, 2016, pp. 483-489. DOI: 10.1109/HSI.2016.7529678.

7. Degottex, Gilles; Ardaillon, Luc; Roebel, Axel. Simple multi frame analysis methods for estimation of amplitude spectral envelope estimation in singing voice. Proc. of IEEE Int. Conf. on Acoustics, Speech and Signal Processing, ICASSP, 20-25 Mar. 2016, Shanghai, China. IEEE, 2016, pp: 4975-4979, DOI: 10.1109/ICASSP.2016. 7472624.

8. Zheng, Shi; Pan, Xuehan; Zhang, Anxue; Jiang, Yansheng; Wang, Wenbing. Estimation of echo amplitude and time delay for OFDM-based ground-penetrating radar. IEEE Geosci. Remote Sensing Lett., Vol. 12, No. 12, pp. 2384-2388, 2015. DOI: 10.1109/LGRS.2015.24782 $\underline{79}$.

9. McKilliam, Robby G.; Pollok, André; Cowley, William; Clarkson, I. Vaughan L.; Quinn, Barry G. Carrier phase and amplitude estimation for phase shift keying using pilots and data. IEEE Trans. Signal Processing, Vol. 62, No. 15, pp. 3976-3989, 2014. DOI: 10.1109/TSP. 2014.2332976.

10. Трифонов, А. П.; Бутейко, В.К. Характеристики совместных оценок параметров сигнала при частичном нарушении условий регулярности. Радиотехника и электроника, Т. 36, № 2, С. 319-327, 1991. 